\title{
Solutions of Sodium in Liquid Ammonia as Described by a Hard Sphere Charge-Dipole Model in the Neutralizing Background
}

\author{
M. F. Golovko and I. A. Protsykevych \\ Statistical Physics Division, Institute for Theoretical Physics of the Ukrainian SSR Academy \\ of Sciences, Lviv, 290005, USSR
}

Z. Naturforsch. 46a, $19-26$ (1991); received October 23, 1990

Dedicated to Dr. Karl Heinzinger on the occasion of his 60 th birthday

The analytic solution of the mean spherical approximation obtained by us previously for the ion-dipole system in a neutralizing background is applied to study the structural, thermodynamic and dielectric properties of sodium-ammonia solutions. It is shown that the structure of ammonia is closepacked and changes little with ion concentration. The shape of the ion-ion distribution function changes from solvation behaviour with Debye-like asymptotics at low ion concentration to density ordering in the metalic region.

\section{Introduction}

Ions in solution have widely been investigated during the last years [1-4]. The simplest model consists of charged hard spheres (ions) and hard spheres possessing dipole moments (solvent molecules). In particular, a set of analytical results has been obtained within the mean spherical approximation (MSA) for the case of equal sizes of the ion and the molecule [5-9], and unequal sizes [10-15]. Patey and coworkers have studied the model using different versions of the hypernetted chain $(\mathrm{HNC})$ approximation $[16,17]$. Recently, besides the dipole moment allowance for the quadrupole moment has been made on the same approximation level $[18,19]$.

The site-site approach $[20,21]$ permits to eliminate the problems of the multipole expansion for the ionmolecule and intermolecular interactions. Monte Carlo (MC) simulation data for the ion-dipole mixture have also become available [22, 23]. The authors of these papers have obtained different results for the ion-ion distribution functions, manifesting the problems in simulating the system at low concentrations. Systematic and consistent molecular dynamics (MD) simulations of aqueous electrolyte solutions were presented in the papers of Heinzinger and coworkers [24]. They

Reprint requests to Prof. Dr. M. F. Golovko, Statistical Physics Division, Institute for Theoretical Physics, Ukrainian SSR Academy of Sciences, Lviv 290005, USSR. were the first who obtained the ion-ion radial distribution functions besides the ion-molecule and moleculemolecule ones and investigated the dependence of these functions on concentration, temperature and pressure.

In this paper we deal with metal $(\mathrm{Na})$ - polar liquid $\left(\mathrm{NH}_{3}\right)$ solutions [25]. We shall apply the two-fluid point of view, in which the solution is considered as consisting of two subsystems, the quantum subsystem of electrons and the classical ion-molecular subsystem. This consideration has been successfully applied for liquid metals $[26,27]$ and metal-electrolyte melts [28-30]. In this approach the ion-molecular system is immersed in a uniform neutralizing background and is considered as the reference fluid which dominates the structure. Allowance for the electron subsystem, at least in the metallic region, similarly to the case of metals or metal-molten salt solutions [31], can be given by means of perturbation theory through the pseudopotential of the electron-molecule interaction and the dielectric function of the electron gas. In the nonmetal region the problem arises to provide consistency of the structure of the solution and the electron subsystem state.

The simplest ion-molecular model with neutralizing background is the ion-dipole one. We have obtained the analytical solution of the MSA for this model [15] and shall apply it here to study solutions of sodium in ammonia. Lithium dissolved in ammonia has been studied by MD simulations by Heinzinger et al. [32]. 
1. The Analytic Expressions for the Two-sort Ion-dipole Model with Neutralizing Background in the MSA

The considered model consists of hard spheres with charge $l Z_{\mathrm{m}}$, density $\varrho_{\mathrm{m}}$ and diameter $\sigma_{\mathrm{m}}$, and hard spheres with point dipole $P_{\mathrm{s}}$, density $\varrho_{\mathrm{s}}$, and diameter $\sigma_{\mathrm{s}}$. These particles are embedded in a uniform neutralizing background of density $\varrho_{\varphi}=\varrho_{\mathrm{m}} Z_{\mathrm{m}}$.

The MSA introduced by Lebowitz and Percus [33] reduces to the solution of the Ornstein-Zernike $(\mathrm{OZ})$ integral equations

$$
\begin{aligned}
h_{i j}\left(X_{1}, X_{2}\right) & =c_{i j}\left(X_{1}, X_{2}\right) \\
& +\sum_{i_{1}=1}^{2} \varrho_{i_{1}} \int \mathrm{d} X_{3} h_{i i_{1}}\left(X_{1}, X_{3}\right) c_{i_{1} j}\left(X_{3}, X_{2}\right),
\end{aligned}
$$

supplemented by the closure for the total correlation functions (TCF) $h_{i j}\left(X_{1}, X_{2}\right)=g_{i j}\left(X_{1}, X_{2}\right)-1$ and the direct correlation function (DCF) $c_{i j}\left(X_{1}, X_{2}\right)$, where

$h_{i j}\left(X_{1}, X_{2}\right)=-1$ for $r_{12}<\sigma_{i j}=\frac{1}{2}\left(\sigma_{i}+\sigma_{j}\right)$,

$c_{i j}\left(X_{1}, X_{2}\right)=-\beta U_{i j}\left(X_{1}, X_{2}\right)$ for $r_{12}>\sigma_{i j}$.

$g_{i j}\left(X_{1}, X_{2}\right)$ are the pair distribution functions (PDF), $U_{i j}\left(X_{1}, X_{2}\right)$ are the electrostatic interaction pair potentials, $\beta=1 / k_{\mathrm{B}} T, X_{1}=\left(\boldsymbol{r}_{1}, \Omega_{1}\right)$ denotes the set of coordinates of particle $1, \Omega_{1}$ is the set of Euler angles necessary to define the orientation of the molecule and $r_{12}$ is the interparticle distance.

The relation (1.2) is exact and (1.3), being approximate, provides the correct asymptotics of the DCF at $r_{12} \rightarrow \infty$.

The TCF and DCF are presented in the orientationinvariant form $[34,35]$

$$
\begin{aligned}
& h_{i j}\left(X_{1}, X_{2}\right)=\sum_{m, n, l} h_{i j}^{m n l}\left(r_{12}\right) \Phi_{00}^{m n l}\left(\Omega_{1}, \Omega_{2}, \Omega_{r_{12}}\right), \\
& c_{i j}\left(X_{1}, X_{2}\right)=\sum_{m, n, l} c_{i j}^{m n l}\left(r_{12}\right) \Phi_{00}^{m n l}\left(\Omega_{1}, \Omega_{2}, \Omega_{r_{12}}\right),
\end{aligned}
$$

where the linear symmetry of thje dipole has been taken into account; $\Omega_{1}, \Omega_{2}, \Omega_{r_{12}}$ are, respectively, the Euler angles specifying the orientation of molecules 1 and 2 with respect to an arbitrary set of axes and of the vector $\boldsymbol{r}_{12}$ joining their centers of mass, and

$$
\begin{gathered}
\Phi_{00}^{m n l}\left(\omega_{1}, \Omega_{2}, \Omega_{3}\right)=\sqrt{(2 m+1)(2 n+1)} \\
\cdot \sum_{\mu, v, \lambda}\left(\begin{array}{lll}
m & n & l \\
\mu & v & i
\end{array}\right) D_{0 \mu}^{m}\left(\Omega_{1}\right) D_{0 v}^{n}\left(\Omega_{2}\right) D_{0 \lambda}^{l}\left(\Omega_{2}\right) .
\end{gathered}
$$

The standard notations for the Wigner 3-j symbols and generalized spherical harmonics have been ap- plied. After integration by orientations and transition to the orientation frame, respectively, to the axis connecting the centers of masses of the particles, (1.1) reduces to the equations in Fourier space

$$
\begin{aligned}
\tilde{H}_{\lambda, i j}^{m n}(\varkappa) & -\tilde{C}_{\lambda, i j}^{m n}(\varkappa) \\
= & \sum_{i_{1}=1}^{2} \sum_{n_{1}=0}^{1}(-1)^{\dot{\lambda}} \varrho_{i_{1}} \tilde{H}_{\lambda, i i_{1}}^{m n_{1}}(\varkappa) \tilde{C}_{\lambda, i_{1} j}^{n_{1} n}(\varkappa),
\end{aligned}
$$

where

$$
\begin{aligned}
& \tilde{H}_{\lambda, i j}^{m n}(\varkappa)=\int_{0}^{\infty} \mathrm{d} r\left[e^{i \times r} J_{\lambda, i j}^{m n}(r)+e^{-i \times r} J_{\lambda, j i}^{n m}(r)\right], \\
& \tilde{C}_{\lambda, i j}^{m n}(\varkappa)=\int_{0}^{\infty} \mathrm{d} r\left[e^{i \times r} S_{\lambda, i j}^{m n}(r)+e^{-i \times r} S_{\lambda, j i}^{n m}(r)\right] .
\end{aligned}
$$

Here the functions $J_{i, i j}^{m n}(r)$ and $S_{i, i j}^{n m}(r)$ can be represented through the coefficients in (1.4):

$$
\begin{aligned}
& J_{\lambda, i j}^{m n}(r)=2 \pi(-1)^{\lambda} \sum_{l}\left(\begin{array}{ccc}
m & n & l \\
\lambda & -\lambda & 0
\end{array}\right) \int_{r}^{\infty} \mathrm{d} t t P_{l}\left(\frac{r}{t}\right) h_{i j}^{m n l}(t), \\
& S_{\lambda, i j}^{m n}(r)=2 \pi(-1)^{\lambda} \sum_{l}\left(\begin{array}{ccc}
m & n & l \\
\lambda & -\lambda & 0
\end{array}\right) \int_{r}^{\infty} \mathrm{d} t t P_{l}\left(\frac{r}{t}\right) c_{i j}^{m n l}(t),
\end{aligned}
$$

where the $P_{l}(r / t)$ are the Legendre polynomials.

The set of (1.6) decouples into two independent equations for $\lambda=0$ and $\lambda=1$, respectively,

$$
\begin{aligned}
& {\left[I+\sqrt{\varrho} * \tilde{H}_{0}(\varkappa) * \sqrt{\varrho}\right] *\left[I-\sqrt{\varrho} * \tilde{C}_{0}(\varkappa) * \sqrt{\varrho}\right]=I,} \\
& {\left[1+\varrho_{\mathrm{s}} \tilde{H}_{1, \mathrm{ss}}^{11}(\varkappa)\right]\left[1-\varrho_{\mathrm{s}} \tilde{C}_{1, \mathrm{ss}}^{11}(\varkappa)\right]=1,}
\end{aligned}
$$

where $I$ is the unit matrix, $\varrho$ is the 3 rd order diagonal matrix of the number densities of the ions and the solvent $\left(\varrho_{3}=\varrho_{\mathrm{s}}\right) ; \tilde{H}_{0}(\%)$ and $\tilde{C}_{0}(\varkappa)$ are the square matrices of order 3 and the asterisk denotes the multiplication of two matrices.

Equation (1.10) has a form similar to the one-component dipole system and reduces to the PercusYevick (PY) equation for hard spheres with effective density $\eta=-b_{2} / 12$, where

$$
b_{2}=\varrho_{\mathrm{s}} \sigma_{\mathrm{s}}^{3} \frac{6 \pi}{\sqrt{30}} \int_{0}^{\infty} \mathrm{d} r \frac{h_{\mathrm{ss}}^{112}(r)}{r} .
$$

Thus [36]

$$
\begin{aligned}
& H_{1, \mathrm{ss}}^{11}(r)=\frac{b_{2}}{2 \pi \varrho_{\mathrm{s}} \sigma_{\mathrm{s}}^{3}} h_{2}^{(\mathrm{PY})}\left(r,-\frac{b_{2}}{12}\right), \\
& C_{1, \mathrm{ss}}^{11}(r)=\frac{b_{2}}{2 \pi \varrho_{\mathrm{s}} \sigma_{\mathrm{s}}^{3}} c_{2}^{(\mathrm{PY})}\left(r,-\frac{b_{2}}{12}\right),
\end{aligned}
$$

where $h_{2}^{(\mathrm{PY})}(r)$ and $c_{2}^{(\mathrm{PY})}(r)$ are the correlation functions of the one-component hard sphere model in the PY approximation. 
In order to solve (1.9), one can apply the BaxterWertheim (BW) method [37, 38], which leads to the representation

$$
\begin{aligned}
& I-\sqrt{\varrho} * \tilde{C}_{0}(\varkappa) * \sqrt{\varrho}=\tilde{Q}(\varkappa) * \tilde{Q}^{\mathrm{T}}(-\varkappa), \\
& {\left[I+\sqrt{\varrho} * \tilde{H}_{0}(\varkappa) \sqrt{\varrho}\right] * \tilde{Q}(\varkappa)=\left[Q^{\mathrm{T}}(-\varkappa)\right]^{-1},}
\end{aligned}
$$

where $\mathrm{T}$ indicates matrix transpose.

By analyzing (1.14), taking account of the closure of the MSA, one represents the functions $Q_{i j}^{m n}(\varkappa)$ as [10]

$$
\begin{aligned}
\tilde{Q}_{i j}^{m n}(\varkappa)=\delta_{i j}^{m n}-\sqrt{\varrho_{i} \varrho_{j}}[ & {\left[\int_{i j i}^{G_{i j}} \mathrm{~d} r q_{i j}^{m n}(r) e^{i \times r}\right.} \\
& \left.-A_{i j}^{m n} \int_{\lambda_{j i}}^{G_{i j}} \mathrm{~d} r e^{(i \times-\mu) r}\right],
\end{aligned}
$$

where $q_{i j}^{m n}(r)=0$ at $r>\sigma_{i j}, \lambda_{j i}=\left(\sigma_{j}-\sigma_{i}\right) / 2$ and $\delta_{i j}^{m n}=$ $\delta_{i j} \delta_{m n}, \delta_{i j}$ being the Kronecker delta function.

The BW functions $Q_{i j}^{m n}(r)$ can be determined by a method given in [15]:

$$
\begin{aligned}
Q_{i j}^{m n}(r)=q_{i j}^{m n}(r)-z_{i} a_{j}^{n}, & \\
q_{i j}^{m n}(r)=\left(r-\sigma_{i j}\right) q_{i j}^{\prime m n} & +\frac{1}{2}\left(r-\sigma_{i j}\right)^{2} q_{i j}^{\prime \prime m n} \\
& +\frac{1}{6}\left(r-\sigma_{i j}\right)^{3} q_{i j}^{\prime \prime \prime m n} .
\end{aligned}
$$

The coefficients of the BW function which define the MSA analytic solution are expressed through the set of seven interaction parameters $M_{i}, v_{i}, \Gamma, B^{10}$ and $b_{2}$. The set of nonlinear equations couple $M_{i}, v_{i}, \Gamma, B^{10}$, $b_{2}$ with the parameters of the ion-ion $\alpha_{0}^{2}=4 \pi \beta e^{2}$ iondipole $\alpha_{1}^{2}=\alpha_{0} \alpha_{2}$, and dipole-dipole $\alpha_{2}^{2}=\frac{4 \pi}{3} \beta \varrho_{\mathrm{s}}^{2}$ inter-
actions:

$$
\begin{aligned}
D_{\Omega} a_{i}^{0}= & 2 \Gamma M_{i} \\
& +\frac{\sigma_{\mathrm{s}}^{2}}{2} \varrho_{\mathrm{s}}\left[\left(\eta_{i}+\Gamma \sigma_{i} v_{i}\right) \sum_{j} \varrho_{j} \sigma_{j} v_{j} M_{j}-\frac{\sigma_{j}}{\beta_{6}} v_{i} B^{10}\right],
\end{aligned}
$$

$M_{i} a_{\mathrm{s}}^{1} D=y_{0} \sigma_{i} v_{i}+\sigma_{\mathrm{s}} \eta_{i}+\frac{\sigma_{\mathrm{s}}}{2} D a_{i}^{0} \sum_{j} \varrho_{j} \sigma_{j} v_{j} M_{j}$, (1.19)

$\alpha_{0}^{2}=\sum_{i} \sum_{m} \varrho_{i}\left(a_{i}^{m}\right)^{2}$,

$\alpha_{0}\left(\alpha_{2}-\alpha_{0} \Lambda^{10}\right) \beta_{6} D=\frac{\sigma_{\mathrm{s}}^{2}}{2} \sum_{i} \varrho_{i} a_{i}^{0} \eta_{i}+a_{\mathrm{s}}^{1} y_{0}$,

$$
\begin{aligned}
\left(D \frac{\beta_{6}^{2}}{\beta_{12}^{2}}\right)^{2}+\varrho_{\mathrm{s}}\left[\left(\alpha_{2}-\alpha_{0} \Lambda^{10}\right) \beta_{6} D\right]^{2} \\
=\frac{\sigma_{\mathrm{s}}^{2}}{4} \varrho_{\mathrm{s}} \sum_{i} \varrho_{i} \eta_{i}^{2}+y_{0}^{2},
\end{aligned}
$$

where

$$
\Lambda^{10}=\frac{\sigma_{\mathrm{s}}^{3}}{12} \sum_{i} \varrho_{i} z_{i}^{*} v_{i}+\frac{\sigma_{\mathrm{s}}^{2}}{4 \beta_{6}} \sum_{i} \varrho_{i} \sigma_{i} v_{i} M_{i} .
$$

The set of (1.18)-(1.22) is solved by numerical methods. In the low ionic density region $\left(\varrho_{m} \rightarrow 0\right)$ one has to neglect the terms which are linear and of higher order in $\varrho_{m}$. Then the interaction parameters can be written in the form

$$
\begin{aligned}
M_{i} & =\frac{z_{i}}{1+\Gamma \sigma_{i}}, \\
v_{i} & =\frac{\alpha_{1}^{2}}{\sqrt{\varepsilon_{\mathrm{W}}}} \cdot \frac{\beta_{12}^{2}}{\sigma_{\mathrm{s}} \beta_{6}+\sigma_{i} \beta_{3}} \cdot \frac{z_{i}}{1+\Gamma \sigma_{i}} \cdot \frac{1+y_{0}}{1+y_{0}+\Gamma \sigma_{\mathrm{s}}}, \\
\Gamma & =\frac{\alpha_{0}}{2 \sqrt{\varepsilon_{\mathrm{W}}}} \sqrt{\varrho_{m}}, \\
B^{10} & =0
\end{aligned}
$$

$b_{2}=b_{2}^{(0)}$,

where $\varepsilon_{\mathbf{W}}=\left(\frac{\beta_{12}^{2} \beta_{3}}{\beta_{6}^{3}}\right)^{2}$ is the Wertheim dielectric constant [36]. The limit of low ion concentration determined by (1.24)-(1.28) is taken as a starting point to iterate the interaction parameters in the nmerical investigation of the considered model.

Let us introduce the functions

$$
G_{i j}^{m n}(r)=H_{i j}^{m n}(r)-E_{i j}^{m n}(r) .
$$

where $E_{i j}^{m n}(r)$ are the functions equal to $H_{i j}^{m n}(r)$ in the hard core region.

To find the functions $G_{i j}^{m n}(r)$, it is necessary to solve the set of convolution integral equations

$$
\begin{gathered}
r G_{i j}^{m n}(r)-\sum_{i_{1}} \sum_{n_{1}} Q_{i_{1}} \int_{\lambda_{j i_{1}}}^{r} \mathrm{~d} t(r-t) G_{i i_{1}}^{m n_{1}}(r-t) Q_{i_{1} j}^{n_{1} n}(t) \\
=\frac{1}{2 \pi}\left[q_{i j}^{\prime m n}+\left(r-\sigma_{i j}\right) q_{i j}^{\prime \prime m n}+\frac{1}{2}\left(r-\sigma_{i j}\right)^{2} q_{i j}^{\prime \prime \prime \prime m}\right] .
\end{gathered}
$$

We can solve (1.30) numerically (for instance by the Perram procedure) or by Laplace transformation.

In the latter case we obtain the following compact expression:

$$
\begin{aligned}
\hat{G}_{i j}^{m n}(s)= & \hat{G}_{i j}^{\mathrm{HS}}(s) \check{b}_{i}^{m} \tilde{b}_{j}^{n} \\
& +\frac{e^{-s \sigma_{i j}}}{2 \pi s}\left\{\frac { \check { v } _ { i } ^ { m } - \frac { 1 } { 2 } } { D _ { r } ( s ) } \left[\frac{\sigma_{\mathrm{s}}^{2}}{D} \varrho_{s}(1-\check{e} \breve{h}) \check{p}_{i}^{m} \check{p}_{j}^{n}\right.\right. \\
& \left.+D_{\Omega}(1-\check{z} \check{p}) \check{h}_{\mathrm{i}}^{\mathrm{m}} \breve{h}_{j}^{n}+D_{\Omega}(\check{z} \breve{h})\left(\check{h}_{i}^{m} \check{p}_{j}^{m}+\check{p}_{i}^{m} \breve{h}_{j}^{n}\right)\right] \\
& \left.-\frac{2}{\varrho_{\mathrm{s}} \sigma_{\mathrm{s}}^{2}} \frac{\check{v}_{i}^{m} \check{v}_{j}^{n}}{1-\check{w} \check{v}}\right\},
\end{aligned}
$$


where the expressions for the 2-component vectors were presented in our previous work [15]. ( $\left.\check{w}^{v} \tilde{v}\right)$ is the vector product.

Replacing in (1.31) the Laplace transform by the Fourier transform yields

$$
\tilde{D}_{i j}^{m n}(\varkappa)=\lim _{s \rightarrow i \propto}\left\{\frac{2 \pi}{s}\left[\hat{G}_{i j}^{m n}(-s)-\hat{G}_{i j}^{m n}(s)\right]\right\} .
$$

Inverting (1.32) to space, the functions $D_{i j}^{m n}(r)$ are

$$
D_{i j}^{m n}(r)= \begin{cases}H_{i j}^{m n}(r), & m=n, \\ G_{i j}^{m n}(r), & m \neq n .\end{cases}
$$

The orientation-invariant coefficients of the TCFs of the considered model in the MSA are

$h_{i j}^{000}(r)=D_{i j}^{00}(r)$,

$h_{i \mathrm{~s}}^{011}(r)=D_{i \mathrm{~s}}^{01}(r)-\frac{1}{r^{2}} \int_{0}^{2} \mathrm{~d} t t D_{i \mathrm{~s}}^{01}(t)$,

$h_{\mathrm{ss}}^{110}(r)=D_{\mathrm{ss}}^{11}(r)-2 H_{1, \mathrm{ss}}^{11}(r)$,

$h_{\mathrm{ss}}^{112}(r)=D_{\mathrm{ss}}^{11}(r)+H_{1, \mathrm{ss}}^{11}(r)$

$$
-\frac{3}{r^{3}} \int_{0}^{2} \mathrm{~d} t t^{2}\left[D_{\mathrm{ss}}^{11}(t)+H_{1, \mathrm{ss}}^{11}(t)\right] .
$$

Finally, we shall present expressions for the excess thermodynamic properties which are based on the analytic solution of the MSA. The electrostatic part of the internal energy for each sort can be written as [15]

$\beta \frac{E_{m}^{\mathrm{cl}}}{N_{m}}=\frac{\alpha_{0}^{2}}{4 \pi} z_{m} \beta_{m}-\frac{\alpha_{1}^{2}}{4 \pi} \varrho_{\mathrm{s}} z_{m} \beta_{6}\left(v_{m}-\frac{\pi}{6} \sum_{j} \varrho_{j} \sigma_{j}^{3} v_{j}\right)$,

$\beta \frac{E_{\mathrm{s}}^{\mathrm{cl}}}{N_{\mathrm{s}}}=-\frac{\alpha_{1}^{2}}{4 \pi} B^{10}-\frac{\alpha_{2}^{2}}{4 \pi} \cdot \frac{b_{2}}{\sigma_{\mathrm{s}}^{3}}$,

where the notations are also given in [15].

The electrostatic part of the chemical potentials is given by [39]

$\beta \mu_{i}^{\mathrm{cl}}=\beta \frac{E_{i}^{\mathrm{cl}}}{N_{i}}-\frac{1}{2} \sum_{j} \varrho_{j}\left[\tilde{c}_{i j}^{\mathrm{R} .000}(\varkappa=0)-\tilde{c}_{i j}^{\mathrm{HS}}(\varkappa=0)\right]$,

where $\tilde{c}_{i j}^{\mathrm{HS}}(\varkappa=0)$ are the Fourier transforms of the DCF at $\varkappa=0$ in the PY approximation and $\tilde{c}_{i j}^{\mathrm{R} .000}(\varkappa=0)$ denotes the regular part of the Fourier transforms of the invariant expansion of the DCF at $\varkappa=0$.

Adelman's dielectric constant can be written as

$\varepsilon=1+\varrho_{\mathrm{s}} \alpha_{2}^{2} \frac{\beta_{12}^{4}}{\beta_{6}^{2}}$, where $\beta_{3 \times 2^{n}}=1+\frac{b_{2}}{3 \times(-2)^{n}}$.

\section{Numerical Calculation and Discussion}

We shall now apply our results for the ion-dipole system in the neutralizing background to describe solutions of sodium metal in liquid ammonia at $235 \mathrm{~K}$. The mole fraction of the completely ionised sodium is

$$
x=\frac{N_{+}}{N_{\mathrm{s}}+N_{+}}
$$

where s stands for solvent. The diameter of the sodium ion is taken from data ionc melts [40], $\sigma_{+}=2.6 \AA$, and that of the ammonia molecule from the compressibility of pure ammonia [25], $\sigma_{\mathrm{s}}=3.0 \AA$. The dipole moment of $\mathrm{NH}_{3}$ is $\varrho_{\mathrm{s}}=1.48 \mathrm{D}$. The molar volume of the mixture is supposed to be additive:

$$
V_{\mu}=x \cdot V_{\mu_{\mathrm{Na}}}+(1-x) V_{\mu_{\mathrm{NH}_{3}}} .
$$

Consider first the interaction parameters $M_{1}, M_{2}$, $v_{1}, v_{2}, \Gamma, B^{10}, b_{2}$, which determine the structural, thermodynamic and dielectric properties of the system. The dependencies of $\Gamma, B^{10}$ and $b_{2}$ on the concentration are presented in Fig. 1. The rapid decreases of $b_{2}$ with increasing concentration means that the approximation $b_{2}=b_{2}^{(0)}$ used in (1.28) is valid at very low concentrations. Also $M_{+}$and $v_{+}$decrease with increasing concentration, while $M_{\mathrm{s}}$ and $v_{\mathrm{s}}$ are zero at $x=0$ and increase slowly.

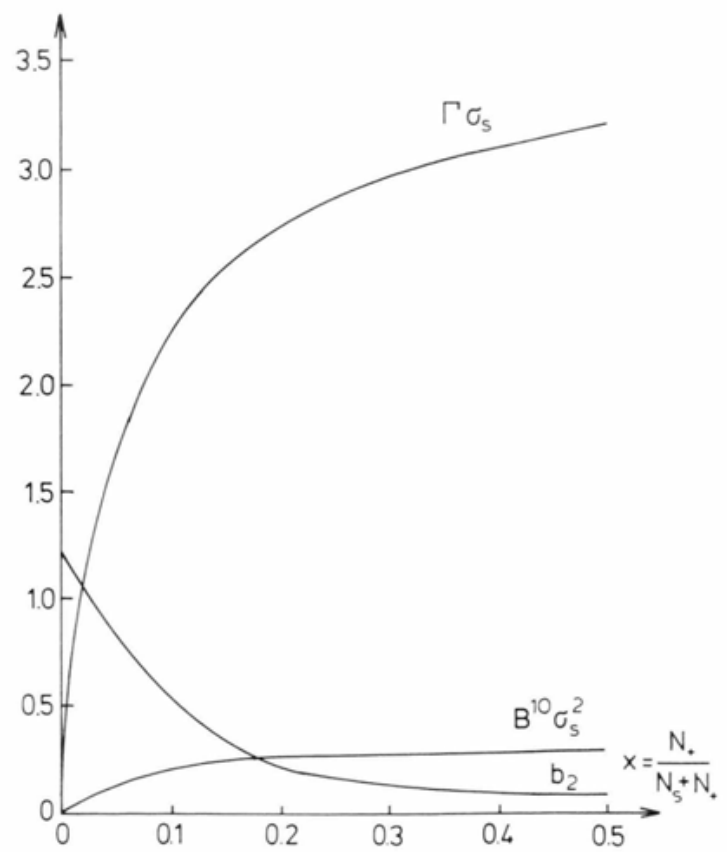

Fig. 1. The MSA parameters $\Gamma, B^{10}$ and $b_{2}$ as a function of $X$. 
By substraction of $g_{i j}^{\mathrm{HS}}(r)$ the screened potentials can be obtained:

$$
G_{i j}\left(X_{1}, X_{2}\right)=g_{i j}\left(X_{1}, X_{2}\right)-g_{i j}^{\mathrm{HS}}\left(r_{12}\right) .
$$

In the following we shall apply the Andersen-Chandler [41] approximation to study the PDFs:

$$
g_{i j}\left(X_{1}, X_{2}\right)=g_{i j}^{\mathrm{HS}}\left(r_{12}\right) e^{G_{i j}\left(X_{1}, X_{2}\right)} .
$$

Contrary to the MSA, this approximation provides positive PDFs for all distances and leads to qualitatively correct functions in a wide range of parameters. The expressions for the PDFs are presented in the orientation-invariant form (1.4). Then the coefficients are

$g_{i j}^{000}(r)=g_{i j}^{\mathrm{HS}}(r) \cdot e^{G_{i j}^{000}(r)}$,

$g_{i \mathrm{~s}}^{000}(r)=g_{i \mathrm{~s}}^{\mathrm{HS}}(r) \cdot \frac{\operatorname{sh} G_{i \mathrm{~s}}^{011}(r)}{G_{i \mathrm{~s}}^{011}(r)}$,

$g_{i \mathrm{~s}}^{011}(r)=g_{i \mathrm{~s}}^{\mathrm{HS}}(r)\left[\frac{\mathrm{ch} G_{i \mathrm{~s}}^{011}(r)}{G_{i \mathrm{~s}}^{011}(r)}-\frac{\mathrm{sh} G_{i \mathrm{~s}}^{011}(r)}{G_{i \mathrm{~s}}^{011}(r)}\right]$,

$g_{\mathrm{ss}}^{000}(r)=\frac{1}{2} g_{\mathrm{ss}}^{\mathrm{HS}}(r) \int_{0}^{\pi} \frac{\mathrm{sh} G_{\mathrm{s}}(R, \vartheta)}{G_{\mathrm{s}}(r, \vartheta)} \sin \vartheta \mathrm{d} \vartheta$,

$g_{\mathrm{ss}}^{110}(r)=\frac{1}{2} g_{\mathrm{ss}}^{\mathrm{HS}}(r) \frac{1}{\sqrt{3}} \int_{0}^{\pi}\left[\operatorname{ch} G_{\mathrm{s}}(r, \vartheta)-\frac{\operatorname{sh} G_{\mathrm{s}}(r, \vartheta)}{G_{\mathrm{s}}(r, \vartheta)}\right]$

$$
\cdot \frac{G^{+}(r) \cos ^{2} \vartheta-G^{-}(r) \sin ^{2} \vartheta}{2 G_{\mathrm{s}}^{2}(r, \vartheta)} \sin \vartheta \mathrm{d} \vartheta
$$

$g_{\mathrm{ss}}^{112}(r)=\sqrt{\frac{5}{6}} g_{\mathrm{ss}}^{\mathrm{HS}}(r) \int_{0}^{\pi}\left[\operatorname{ch} G_{\mathrm{s}}(r, \vartheta)-\frac{\operatorname{sh} G_{\mathrm{s}}(r, \vartheta)}{G_{\mathrm{s}}(r, \vartheta)}\right]$

where

$$
\frac{2 G^{+}(r) \cos ^{2} \vartheta+G^{-}(r) \sin ^{2} \vartheta}{2 G_{\mathrm{s}}(r, \vartheta)} \sin \vartheta \mathrm{d} \vartheta,
$$

$$
\begin{aligned}
& G_{\mathrm{s}}^{2}(r, \vartheta)=\left[G^{+}(r)\right]^{2} \cos ^{2} \vartheta+\left[G^{-}(r)\right]^{2} \sin ^{2} \vartheta, \\
& G^{+}(r)=2 G_{\mathrm{ss}}^{112}(r)+G_{\mathrm{ss}}^{110}(r), \\
& G^{-}(r)=G_{\mathrm{ss}}^{110}(r)-G_{\mathrm{ss}}^{112}(r) .
\end{aligned}
$$

Let us discuss the functions $g_{\mathrm{ss}}^{000}(r)$ and $g_{++}^{000}(r) \cdot g_{\mathrm{ss}}^{000}(r)$ equals $g_{\mathrm{NN}}(r)$ and is the ammonia-ammonia RDF while $g_{++}^{000}(r)$ is the ion-ion RDF.

The curve $g_{\mathrm{NN}}(r)$ for pure ammonia determined by (2.3) is presented in Figure 2. The curves from X-ray measurements [42] and MD simulation [32] are also

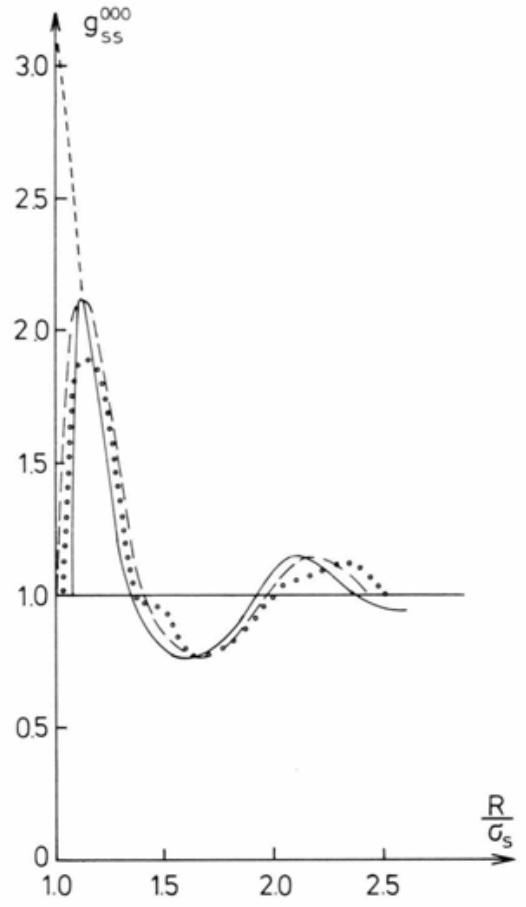

Fig. 2. The nitrogen-nitrogen PDF $g_{\mathrm{ss}}^{000}(r)$ for $x=0.0001$ as a function of the distance between the dipoles. 000 : experimental, _- : MD, - : theory, --- : uncorrected theory.

presented for comparison. It can be seen that our uncorrected theory deviates from the experiment at small distances. It is necessary to allow for the softness of the intermolecular interactions at these distances. In order to do that, the procedure of Weeks, Chandler and Andersen [43] has been applied. The potential $U_{\mathrm{ss}}\left(r, \Omega_{1}, \Omega_{2}\right)$ used already in [32] has been decomposed as follows:

$$
\begin{aligned}
& \varphi_{\mathrm{NN}}(r) \\
& =\left\{\begin{array}{ll}
U_{\mathrm{ss}}\left(r, \Omega_{1}^{p r}, \Omega_{2}^{p r}\right)-U_{\mathrm{ss}}\left(r_{\min }, \Omega_{1}^{p r}, \Omega_{2}^{p r}\right) & \text { for } r<r_{\min } \\
0 & \text { for } r>r_{\min }
\end{array},\right.
\end{aligned}
$$

where $U_{\mathrm{ss}}\left(r_{\min }, \Omega_{1}^{p r}, \Omega_{2}^{p r}\right)$ is the potential minimum for preferable orientations $\Omega_{1}^{p r}$ and $\Omega_{2}^{p r}$.

The allowance of softness in accord to (2.4) leads to the substitution of $g_{\mathrm{ss}}^{\mathrm{HS}}(r)$ in $(2.3)$ by $e^{-\beta \varphi_{N \mathrm{~N}}(r)} g_{i j}^{\mathrm{HS}}(r)$. As can be seen in Fig. 2 this provides the correct first maximum of $g_{\mathrm{NN}}(r)$. The theory and experiment show the first maximum at $3.4 \AA$. However, similarly to the MD data, we did not get the pronounced shoulder at $\sim 3.7 \AA$, manifesting that the model is not safisfactory. 
As already mentioned by Heinzinger et al. [32] the $g_{\mathrm{NN}}(r)$ behaviour indicates a close-packed structure of ammonia. Therefore, contrarily to the function $g_{00}(r)$ for water and aqueous solutions [44], the structure of ammonia and its solutions is determined mainly by density ordering. The contribution of electrostatic interactions and hydrogen bonding is considerably weaker than in water. Our study shows a comparatively weak sensitivity of $g_{i j}^{000}(r)$ on the ion concentrations in a wide range. More sensitive are the harmonics $g_{\mathrm{ss}}^{110}(r)$ and $g_{\mathrm{ss}}^{112}(r)$. The function $g_{\mathrm{ss}}^{112}(r)$ at two different concentrations corresponding to the nonmetallic and metallic regions is presented in Figure 3.

The ion-ion function $g_{++}(r)$ is especially sensitive on the concentration. This can be seen in Fig. 4 for the nonmetallic and metallic regions. Similarly to electrolyte solutions [3], at low ion concentration (nonmetallic region) $g_{++}(r)$ has Debye-like asymptotics. The allowance for solvent is manifested by the oscillations around the asymptotic behaviour due to solvation. At higher concentrations (metallic region) the asymptotic behaviour is absent. Now the oscillations are around the abscissa axis and their amplitude increases. The function $g_{++}(r)$ becomes similar to the one in the case of simple liquids and liquid metals [26, 27]. One has to mention the more structured form of $g_{++}(r)$ than the one for the aforementioned systems.

As for $g_{i j}(r, \Omega)$, our preliminary calculations show that it has a first maximum which corresponds to the orientation $\vartheta_{\mathrm{s}}=0$, manifesting the presence of solvation shells. At increasing concentrations the structure becomes more smooth. However the dominating orientation $\vartheta_{\mathrm{s}}=0$ preserves.

Finally we should like to discuss some thermodynamic and dielectric properties of the model considered. The dielectric constant is determined by (1.41). Its dependence on the concentration is presented in Figure 5. Similarly to the case of electrolyte solutions [2], the dielectric constant decreases with increasing concentration of ions.

The electrostatic part of the internal energy for each short is given by (1.38)-(1.39). Then the total electrostatic part of the internal energy of the system can be written as

$$
\beta \frac{E^{\mathrm{el}}}{V}=\frac{\alpha_{0}^{2}}{2 \pi} \sum_{i} \varrho_{i} z_{i} B_{i}-\frac{\alpha_{1}^{2}}{2 \pi} \varrho_{\mathrm{s}} B^{10}-\frac{\alpha_{2}^{2}}{2 \pi} \frac{\varrho_{\mathrm{s}}}{G_{\mathrm{s}}^{3}} b_{2},
$$

where the first term corresponds to the ion-ion contribution $E_{\mathrm{ii}}^{\mathrm{cl}}$, the second term is ion-dipole one $E_{\mathrm{id}}^{\mathrm{el}}$ and

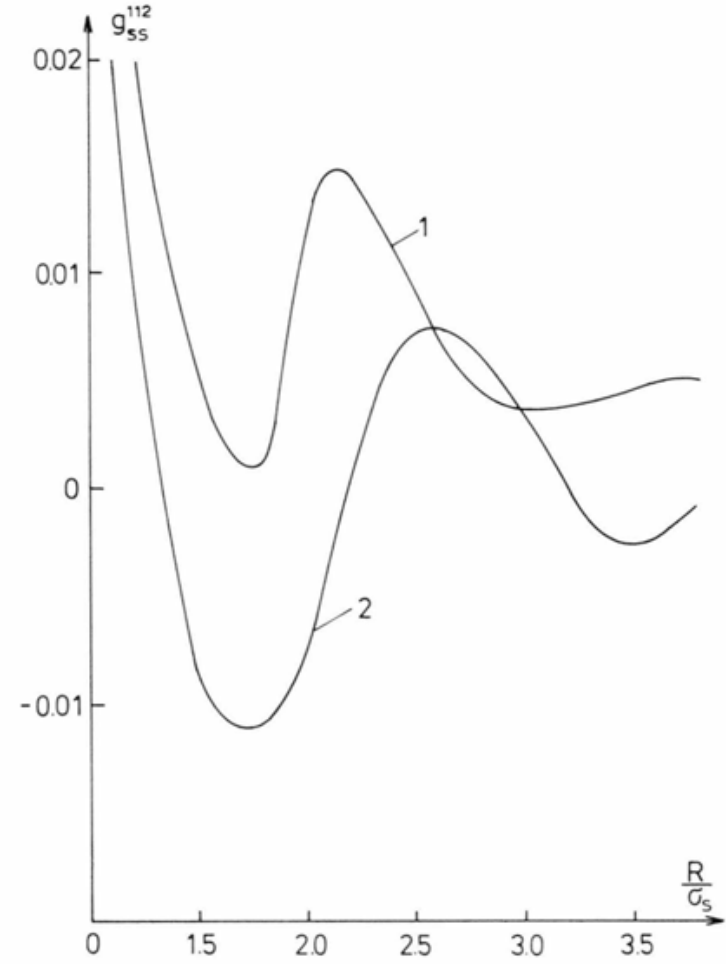

Fig. 3. The PDF $g_{\mathrm{ss}}^{112}(r)$ as a function of the distance between the dipoles for $1: x=0.005$ and $2: x=0.1$.

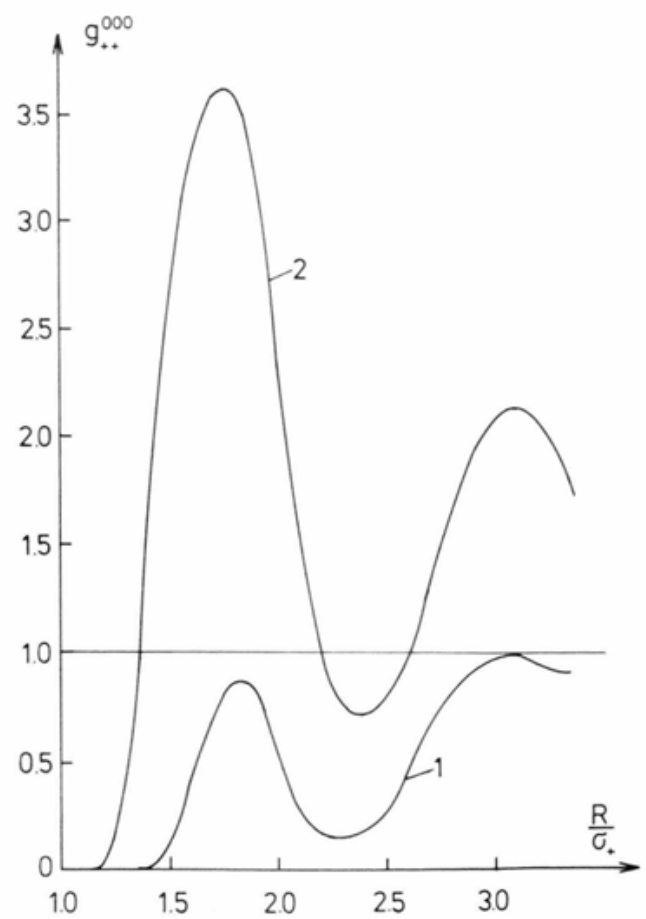

Fig. 4. The ion-ion PDF $g_{\mathrm{ss}}^{110}(r)$ as a function of the distance between the ions for 1: $x=0.005$ and $2: x=0.1$. 


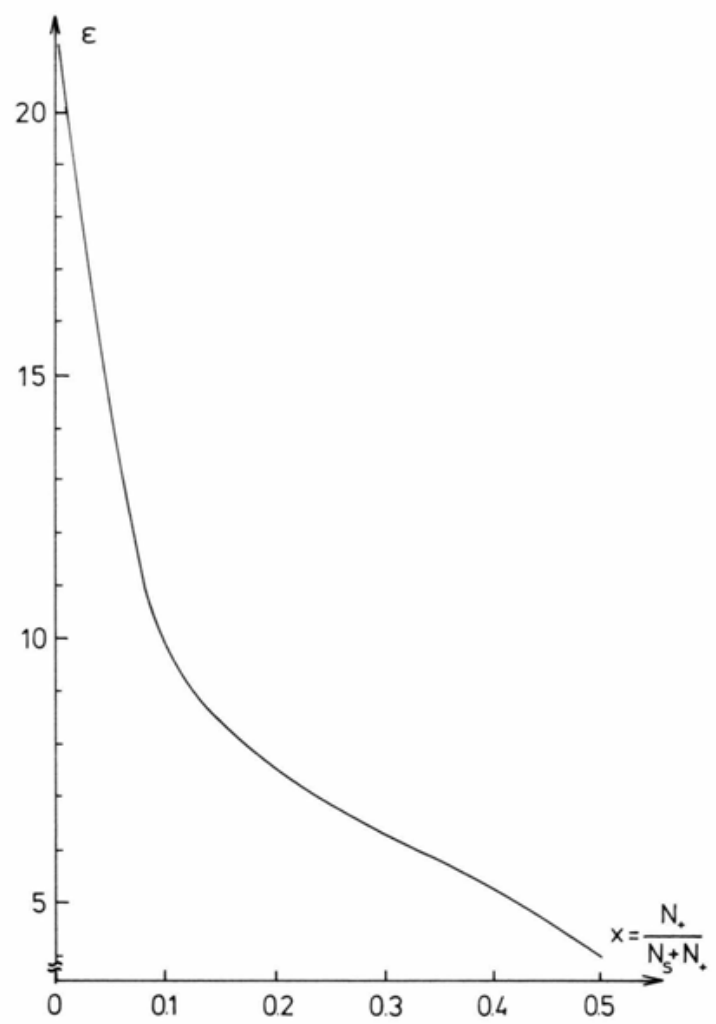

Fig. 5. The dielectric function as a function of ionic concentration.

the third term is dipole-dipole one $E_{\mathrm{dd}}^{\mathrm{el}}$. These contributions dependent on the ion concentration as presented in Figure 6. The ion-ion contribution $E_{\mathrm{ii}}^{\mathrm{el}}$ is the most important one, and its absolute value increases with ion concentration. The absolute value of $E_{\mathrm{dd}}^{\mathrm{cl}}$ decreases with ion concentration while the ion-dipole contribution $E_{\mathrm{id}}^{\mathrm{el}}$ has a maximum at $x=0.23$. At higher concentrations the latter decreases to zero.

One has to note that these properties are considered as the specific features of the model. One has to allow for the electron subsystem when considering a realistic metal-ammonia solution. The situation is very similar to the case of metal-molten salt solutions [28, 29]. Anyway, the ion-molecular model can be considered as a reference fluid for the real metal-ammonia solutions.

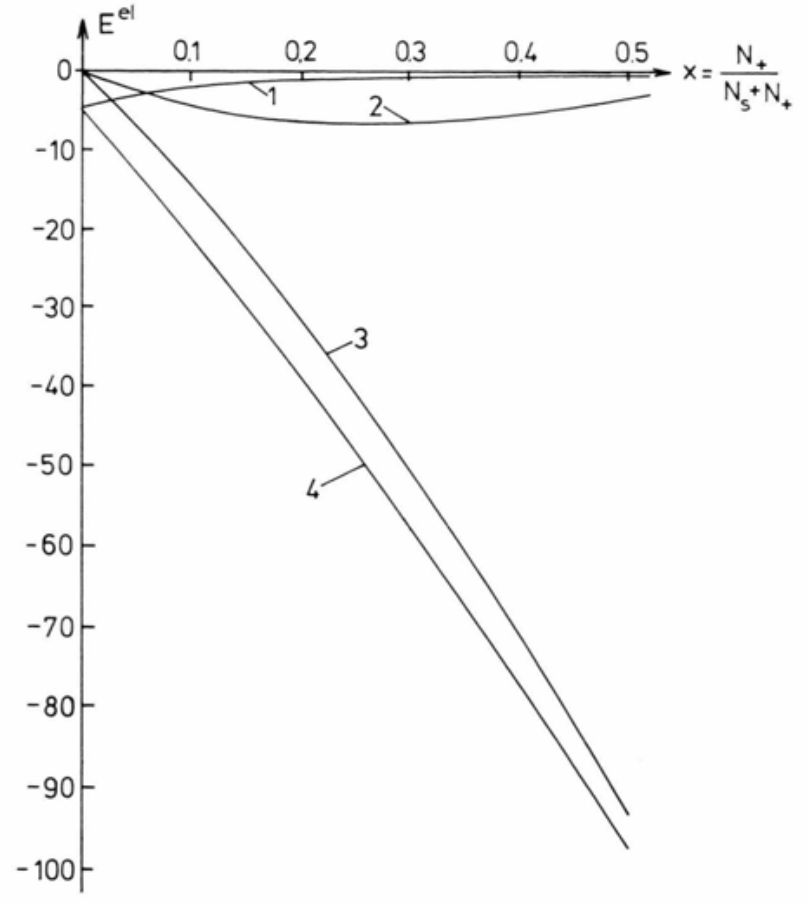

Fig. 6. The electrostatic part of the internal energy as a function of an ionic concentration. 1: ion-ion contribution, 2 : iondipole contribution, 3: dipole-dipole contribution, 4 : total contribution.

\section{Conclusion}

Here we have used the analytical solution of the MSA obtained in [15] for the ion-dipole model in a neutralizing background for the description of the structural, thermodynamic and dielectric properties of solutions of sodium in ammonia. The results show that the structure of ammonia is close-packed and changes little with ion concentration. The form of the ion-ion distribution function changes fromt the solvation behaviour with Debye-like asymptotics at low ion concentration to density ordering in the metallic region. However, for the description of the atom-atom correlation functions $g_{\mathrm{NH}}(r), g_{\mathrm{HH}}(r)$ and $g_{\mathrm{HNa}}(r)$ the present model should be improved. Particularly, the site-site approach for ion-molecular systems in the neutralizing background will be useful for this aim. 
[1] I. R. Yukhnovsky and M. F. Golovko, The Statistical Theory of Classical Equilibrium Systems, Naukowa Dumka, Kiev 1980 (in Russian).

[2] L. Blum and F. Vericat, in: The Chemical Physics of Solvation (R. R. Dogonadze, E. Kalman, A. A. Kornyshev, and J. Ulstrup, eds.), Elsevier, Amsterdam 1985, Chapter 5, p. 143.

[3] M. F. Golovko and I. R. Yukhnovsky, ibid. Chapter 6, p. 207.

[4] K. Heinzinger and G. Palinskas, ibid. Chapter 8, p. 313.

[5] L. Blum, Chem. Phys. Lett. 26, 200 (1974); J. Chem. Phys. 61, 2129 (1974).

[6] S. A. Adelman and J. M. Deutch, J. Chem. Phys. 60, 3935 (1974).

[7] F. Vericat and L. Blum, J. Stat. Phys. 22, 593 (1980).

[8] W. Perez-Hernandez, and L. Blum, J. Stat. Phys. 24, 451 (1981).

[9] M. F. Golovko, The statistical theory of mixed ionmolecular systems, Doctoral Thesis, Institute for Theoretical Physics, Kiev 1979 (in Russian).

[10] L. Blum, J. Stat. Phys. 18, 451 (1978).

[11] L. Blum and D. Q. Wei, J. Chem. Phys. 87, 555 (1987).

[12] D. Q. Wei and L. Blum, J. Chem. Phys. 87, 2999 (1987).

[13] M. F. Golovko and I. A. Protsykevich, Chem. Phys. Lett. 142, 463 (1987).

[14] M. F. Golovko and I. A. Protsykevich, The screened potentials of asymmetric ion-dipole systems, Preprint ITP-86-168 R, Institute for Theoretical Physics, Kiev 1987 (in Russian).

[15] M. F. Golovko and I. A. Protsykevich, J. Stat. Phys. (USA) 54, 707 (1989).

[16] D. Levesque, J. Weis, and G. N. Patey, J. Chem. Phys. 72, 1887 (1980).

[17] P. G. Kusalik and G. N. Patey, J. Chem. Phys. 88, 7715 (1988).

[18] J. M. Caillol, P. Levesque, J. J. Weis, P. G. Kusalik, and G. N. Patey, Mol. Phys. 89, 4994 (1988).

[19] W. Dong, M. L. Rosinberg, A. Perera, and G. N. Patey, J. Chem. Phys. 89, 4994 (1988).

[20] M. F. Golovko and Yu. V. Kalyuzhnij, Mol. Phys. 66, 375 (1989).
[21] M. F. Golovko and Yu. V. Kalyuzhnij, Mol. Phys. 68, 1239 (1989).

[22] K. Yu. Chan, K. E. Gubbins, D. Henderson, and L. Blum, Mol. Phys. 66, 299 (1989).

[23] J. M. Caillol, D. Levesque, and J. J. Weis, Mol. Phys. 69, 199 (1990).

[24] K. Heinzinger, in: Computer Modelling of Fluids, Polymers and Solids (R. Catlow et al., eds.), Kluver Academic Publisher, Dordrecht 1990, p. 357.

[25] J. S. Thompson, Electrons in Liquid Ammonia, Clarendon Press, Oxford 1976.

[26] H.B. Singh and A. Holz, Phys. Rev. A 28, 1108 (1983).

[27] S. K. Lai, Phys. Rev. A 31, 3886 (1986).

[28] G. Chabrier and J.-P. Hansen, Mol. Phys. 50, 901 (1983).

[29] G. Chabrier, G. Senatore, and M. P. Tosi, Nuovo Cim. 1D, 409 (1982).

[30] M. F. Golovko and I. A. Protsykevich, Ukr. Phys. J. 33, 1367 (1988).

[31] G. Chabrier and J.-P. Hansen, Mol. Phys. 59, 1345 (1986).

[32] S. V. Hannongbua, T. Ishida, E. Spohr, and K. Heinzinger, Z. Naturforsch. 43a, 572 (1988).

[33] J. L. Lebowitz and J. K. Percus, Phys. Rev. 144, 251 (1966).

[34] L. Blum and A. J. Toruella, J. Chem. Phys. 56, 303 (1972).

[35] L. Blum, J. Chem. Phys. 57, 1862 (1972); 58, 3295 (1973).

[36] M. S. Wertheim, J. Chem. Phys. 55, 4291 (1971).

[37] R. J. Baxter, Aust. J. Phys. 21, 563 (1968); J. Chem. Phys. 52, 4559 (1970).

[38] M. S. Wertheim, J. Math. Phys. 5, 643 (1964).

[39] J. S. Høye and G. Stell, J. Chem. Phys. 67, 439 (1977).

[40] M. C. Abramo, C. Caccamo, G. Pizzimenti, M. Parrinello, and M. P. Tosi, J. Chem. Phys. 68, 2889 (1978).

[41] H. C. Andersen and D. Chandler, J. Chem. Phys. 57, 1918 (1972).

[42] A. H. Narten, J. Chem. Phys. 66, 3117 (1977).

[43] J. D. Weeks, D. Chandler, and H. C. Andersen, J. Chem. Phys. 54, 5237 (1971).

[44] M. F. Golovko, Yu. V. Kalyuzhnij, and K. Heinzinger, Z. Naturforsch. (in press). 